\title{
DiffServ over WiMAX network simulation testbed
}

\author{
Oumarou HALIDOU ${ }^{1}$ and Radouane MRABET $^{2}$ \\ ${ }^{1,2}$ Mohammed V University - Souissi, National High School for Computer Sciences and Systems \\ Analysis, Rabat, Morocco \\ E-mail: 'oumarhalid@yahoo.fr, ${ }^{2}$ mrabet@ensias.ma
}

\begin{abstract}
The broadband wireless IEEE 802.16 technology, known as WiMax transmission, is one of the first systems designed as multi service integrating QoS management from the beginning. Its wide deployment through the Internet has attracted great interest from researchers and industrialists. WiMAX has defined five classes of service as well as their requirements. However, the standard has not specified the different scheduling and queue management algorithms to use. In this paper, we are presenting simulations of a WiMAX network with several scenarios that we have defined under the NS simulator, involving different data flow to measure the quality of the service offered.
\end{abstract}

Keywords: WiMAX, DiffServ QoS, scheduling, queue, NS.

\section{INTRODUCTION}

The traffic on internet networks has increased significantly in recent years not only in volume, due to the larger number of users and the emergence of new applications, but also in kind, through the integration of several types of information that have different pressure on the same media. The All-IP concept whose aim is to migrate all traditional services to the IP technology is becoming a reality. The user is increasingly looking for a broadband wireless environment, omnipresent, and especially at low cost, that guarantees him the connectivity and the Internet access wherever he is and at any time. It has resulted into a development and a wide propagation of smart mobile terminals such as PDAs, smart phones and tablets.

It is in that context that IEEE has developed a new standard, the 802.16 , commonly called WiMAX. It allows companies or individuals wireless connection over long distances at high speed. WiMAX is an adequate response to some remote or not easy to get to rural areas, which are currently without access to broadband Internet for cost reasons. The announced ranges are $50 \mathrm{~km}$ with a speed of $70 \mathrm{Mbits}$ [1].

Like the IETF DiffServ architecture, WiMAX provides a differentiation of the types of traffic according to the priority and requirements of each.
Thus the IEEE 802.16-2004 standard defines four classes that are: Unsolicited Grant Service (UGS), Real-Time Polling Service (rtPS), Non- Real -Time Polling Service (nrtPS) and the Best Effort (BE) Service. The amendment IEEE 802.16e has added a fifth type of QoS service to the standard, called Extended Real Time Polling Service (ertPS).

However, the integration of the QoS is limited to the specification of the service classes ensuring the differentiation. The standard does not describe a scheduling system for uplink and downlink. So several researches have been conducted in that direction, in order to develop an efficient system of traffic management on 802.16 networks that allows a wide deployment through Internet.

In [2], we have proposed a multi-layered architecture based on the 802.16 standard that ensures a service quality through Internet. Thus, to provide mobile stations (MS) an end to end QoS we have decided to combine the 802.16 standard with the DiffServ architecture model defined by IETF. This consideration of the QoS at both layers will allow a large-scale deployment of services with high QoS constraints through Internet. To automate the management of such architecture and the process of negotiating services contracts, we have developed components based on web services as well as policy rules via the COPS protocol and its extensions. Each layer having its specific 
characteristics and constraints, we have defined rules to map the QoS parameters of the different layers. So we have chosen the adequate scheduling algorithms to ensure the services differentiation.

In this paper, we will present series of simulation tests of DiffServ architecture in WiMAX. In these tests carried on with NS, we are interested by the scheduling and queue management mechanisms. Comparisons will also be made with the results of experimentation, including those conducted by the project TF-TANT [3].

\section{SIMULATIONS AND RESULTS}

In this section, we are presenting various simulations on a number of architectures using the NS (Network Simulator). The objective being to study the interconnection of WiMAX cells to the IP networks implementing DiffServ protocol. We are using the NS 2.9 version on which we have installed the WiMAX module developed by the High Speed Network Technology Group of NIST (National Institute of Standards and Technology) [4]. This implementation involves both physical and MAC layers. The module being developed is based on the IEEE 802.16-2004 and IEEE 802.16e2005 mobile extension.

\section{A. Scenario $n^{\circ} 1$ : Comparison of UGS traffic using $P Q$ and $W F Q$ scheduling algorithms}

The scenario $\mathrm{n}^{\circ} 1$ as illustrated in figure 1, consists of a WiMAX cell where we have a base station managing a certain number of client workstations on which CRB applications are run. In this architecture, we focus on the choice of PQ and WFQ scheduling algorithms.

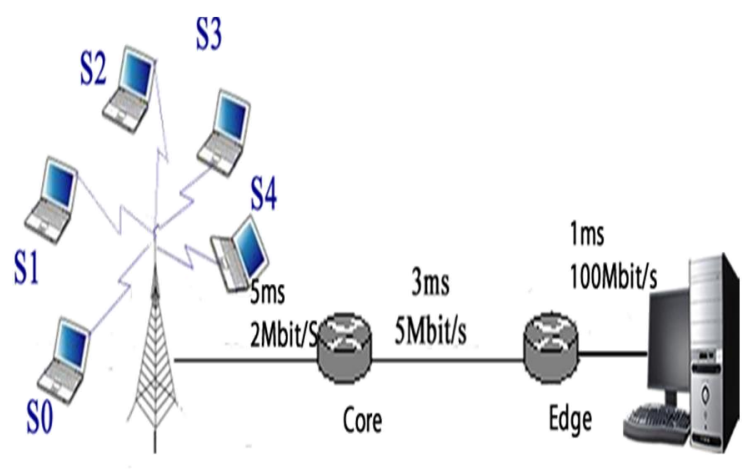

Fig. 1. Architecture scenario $n^{\circ} 1$

For WFQ, it was necessary to find suitable configuration settings. With this algorithm, the flow of $\mathrm{Sr}$ service depends on the weight given to the queue wq and on the packet length $\mathrm{L}$.
In the PriorityQueueing, no configuration is necessary; a packet from the priority queue is served as soon as the interface finishes to transmit the current packet. Therefore, the delay observed by a UGS package is limited to the transmission time of a packet of the MTU seized.

The results of the comparison are presented in figure 2. It shows the delay observed according to the size of the UGS packets. With PQ, the increase in the delay is only matching with the increase of the transmission time related to the packets size. On the other hand, WFQ due to the consideration of the size in the calculation of the time tag, the delay observed by a small packet and a large packet is multiplied by 4 . There is a direct relationship between the time and the size of the EF packets regardless of the scheduler used. However, the results show that a wEF oversizing (the weight given to the $\mathrm{EF}$ queue) decreases the delay but increases the rate of packets loss. Similar results have been proven in the case of a DiffServ network in RFC2598 [5].

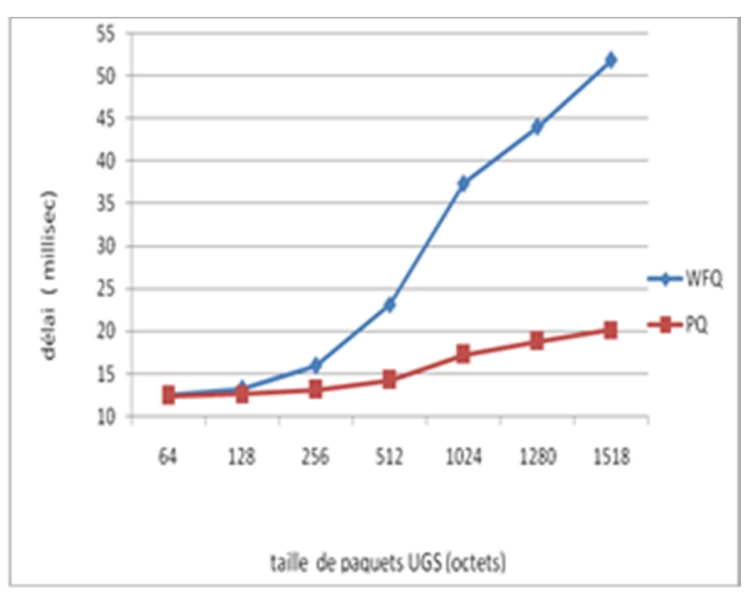

Fig. 2. Comparison of $P Q \& W F Q$

It is a reputation based dynamic and weighted transitive trust management system based on DSR protocol. Trust in this mechanism is established through direct and indirect observations [2].

\section{B. Scenario $n^{\circ}$ 2: behaviour of both $W F Q$ and $P Q$ schedulers with traffics sensitive to the delay and to the jitter}

This scenario is based on a study conducted as part of the project TF-TANT. That study aimed at analysing the performance of a real DiffServ wireline network. We are taking up some of their measurements in an architecture consisting of two WiMAX networks (or cells), each controlled by a base station and interconnected by a wired link illustrated with the figure below. The difference 
with the scenario of TF-TANT project is that instead of wired stations, we put WiMAX clients.

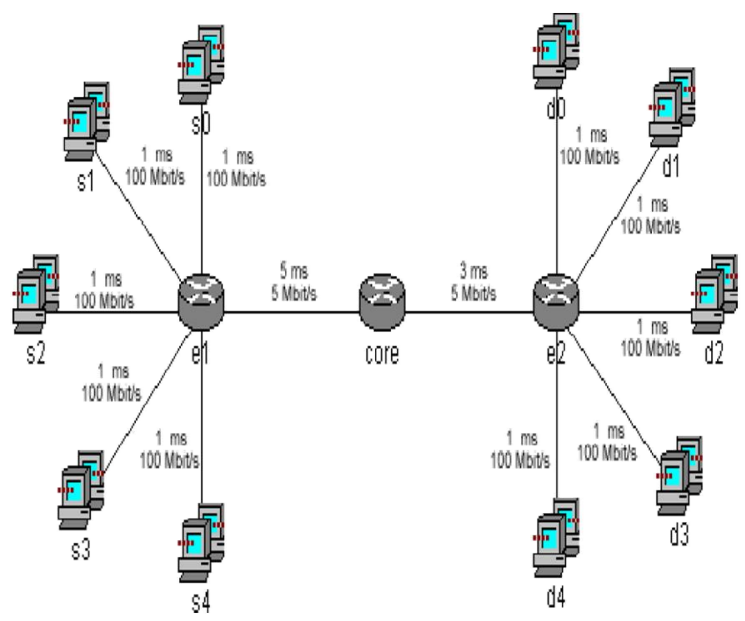

Fig. 3. Project test architecture TF-TANT [3]

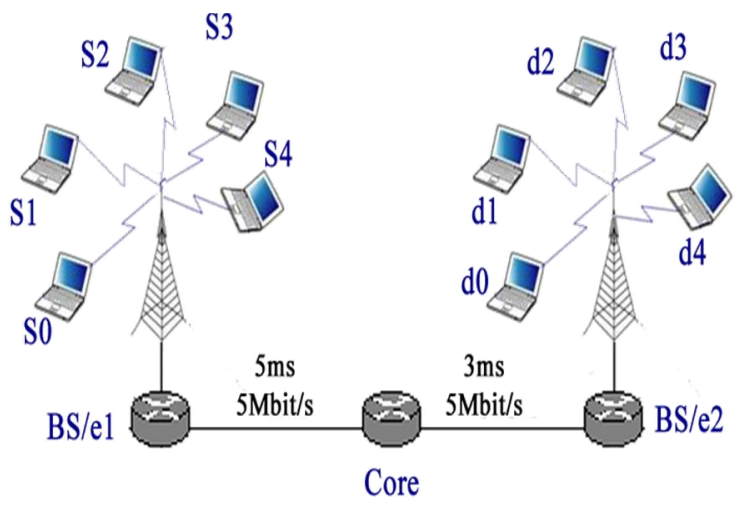

Fig. 4. Architecture scenario $n^{\circ} 2$

In the experiments of TF-TANT, a test bench has been set up to study the behaviour of both WFQ and PQ schedulers with traffics sensitive to the delay and to the jitter. The following tests are considered:

\section{a) Test 1: Relationship between the ef service rate and the average OWD}

In this test, there is a diffServ domain, the edges routers (e1 and e2) are configured with the WFQ scheduler and two queues to support the service classes EF and BE. The EF traffic is generated by a CRB application with a constant speed of $300 \mathrm{kbps}$ whose parameters are described in the TCS table (Traffic Conditioning Specification) below. To avoid synchronization problems, the background traffic is generated by several CRB sources, whose speeds are chosen randomly within the interval [10kbps $100 \mathrm{kbps}]$ and the start time within [0s 5s]. The size of the BE packets has always been 1000 bytes.
In our simulation, the CRB client application has initially been supported at the WiMAX level by a UGS services flow which will then be mapped in the PHB EF within the network by the base station.

Table I: TCS for E1 Interface to Core

\begin{tabular}{|c|l|l|l|l|l|}
\hline $\begin{array}{c}\text { Service } \\
\text { Class }\end{array}$ & $\begin{array}{l}\text { DS } \\
\text { CP }\end{array}$ & $\begin{array}{c}\text { Throug- } \\
\text { hput }\end{array}$ & $\begin{array}{c}\text { Traffic } \\
\text { throughput } \\
\text { rate }\end{array}$ & Meter & Dropper \\
\hline EF & 46 & $\begin{array}{l}\text { Variable } \\
\text { depends } \\
\text { on STAR }\end{array}$ & 300 Kbps & $\begin{array}{l}\text { Token } \\
\text { bucke-t } \\
\text { CIR } \\
300 \\
\text { kbps } \\
\text { CBS 1 } \\
\text { packet }\end{array}$ & $\begin{array}{l}\text { Delete } \\
\text { non- } \\
\text { complia } \\
\text { nt } \\
\text { packets }\end{array}$ \\
& & & & & \\
\hline BE & 0 & $\begin{array}{l}\text { Remainin } \\
\mathrm{g}\end{array}$ & 2.3 Mbps & $\begin{array}{l}\text { Eliminat } \\
\text { ion on } \\
\text { threshol } \\
\text { d }\end{array}$ \\
\hline
\end{tabular}

In RFC2598 the STAR (Service -To- ArrivalRatio) is defined as follows: $\mathrm{Sr} * \mathrm{lr}=\mathrm{Ar} * \mathrm{STAR}$

With: Sr the weight of the service in the WFQ discipline, $1 \mathrm{r}$ the line speed and Ar the speed at which the EF traffic is generated.

According to the settings defined in TF-TANT, the simulation was performed for 50 seconds. For different STAR values and EF packets size the average OWD [6] (One Way Delay) was measured. The figure 5 is showing the different curves of the experiment (Fig. 5a) and of our simulation (Fig. $5 \mathrm{~b}$ ). The results of the simulation show a stable OWD, while the experiment of the real network shows a rise of OWD. The Increase of the value of STAR significantly reduces the OWD, especially for larger packets in the real network. These differences are due to the fact that in the simulated network a QoS is first applied to the UGS traffic with a strict priority at the base station, thus regulating the traffic at the entrance of the router e1.

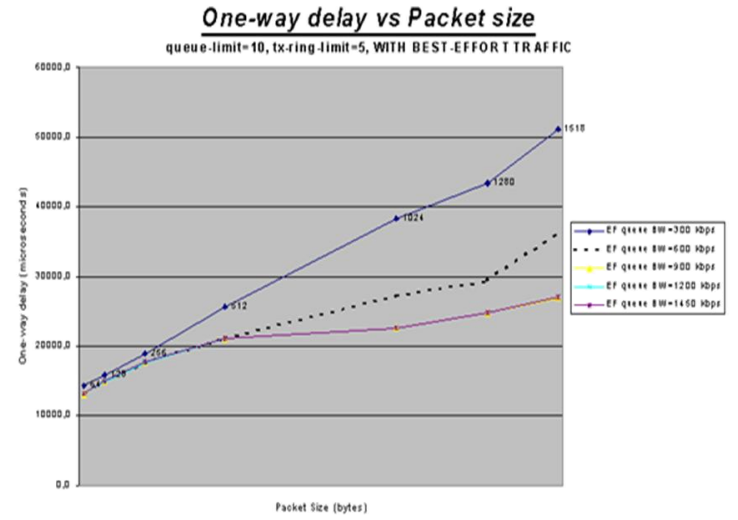

Fig. 5. $\quad a:$ Relationship between the ef service rate and the average OWD compared with WFQ packets size: experimentation [3] 
O. Halidou and R. Mrabet / International Journal of Computer Networks and Communications Security, 1 (6), November 2013

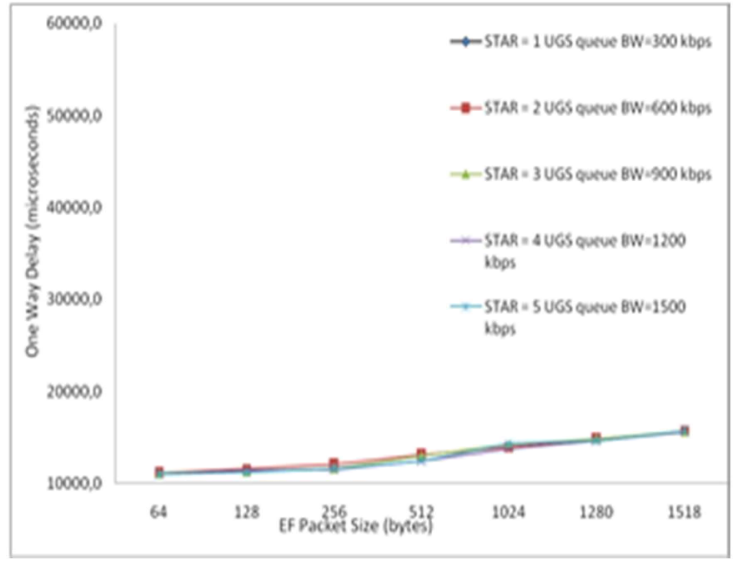

Fig. 5. $\quad$ : Relationship between the ef service rate and the average OWD compared with WFQ packets size : Simulation.

b) Test 2: Impact of the BE packets size on the OWD of EF traffic with the $P Q$ scheduler

In this test, the scheduler PQ is used with two queues for the EF and BE traffic. The EF traffic is generated by a CRB application with a constant speed of $300 \mathrm{kbps}$, as for the background traffic it is generated by several CRB sources whose speeds are chosen randomly within the interval $[10 \mathrm{kbps}$ $100 \mathrm{kbps}]$ and the start time within [0s 5s]. The BE packets size fluctuate between 100 and 1450 bytes in accordance with TF-TANT experiment whose e1 interface configuration to core is described in Table II.

Table II: TCS for E1 Interface to Core

\begin{tabular}{|c|l|l|l|l|l|}
\hline $\begin{array}{c}\text { Servi } \\
\text { ce } \\
\text { Class }\end{array}$ & $\begin{array}{c}\text { DSC } \\
\mathbf{P}\end{array}$ & $\begin{array}{l}\text { Throug- } \\
\text { hput }\end{array}$ & $\begin{array}{c}\text { Traffic } \\
\text { throughpu } \\
\text { t rate }\end{array}$ & Meter & $\begin{array}{l}\text { Droppe } \\
\mathbf{r}\end{array}$ \\
\hline $\mathbf{E F}$ & 46 & $\begin{array}{l}300 \\
\text { Kbps }\end{array}$ & 300 Kbps & $\begin{array}{l}\text { Token } \\
\text { bucket } \\
\text { CIR } \\
300 \\
\text { kbps } \\
\text { CBS 1 } \\
\text { packet }\end{array}$ & $\begin{array}{l}\text { Delete } \\
\text { non- } \\
\text { complia } \\
\text { nt } \\
\text { packets }\end{array}$ \\
\hline BE & 0 & $\begin{array}{l}\text { Remaini } \\
\text { ng }\end{array}$ & 2.3 Mbps & & $\begin{array}{l}\text { Eliminat } \\
\text { ion on } \\
\text { threshol } \\
\text { d }\end{array}$ \\
\hline
\end{tabular}

The experiment was conducted for 50 seconds, with the aim of studying the impact of the size of the BE packets on the One-Way-Delay and on the IP packets delay variation of (IPDV) [7]. Figures 6 and 7 show the different curves.
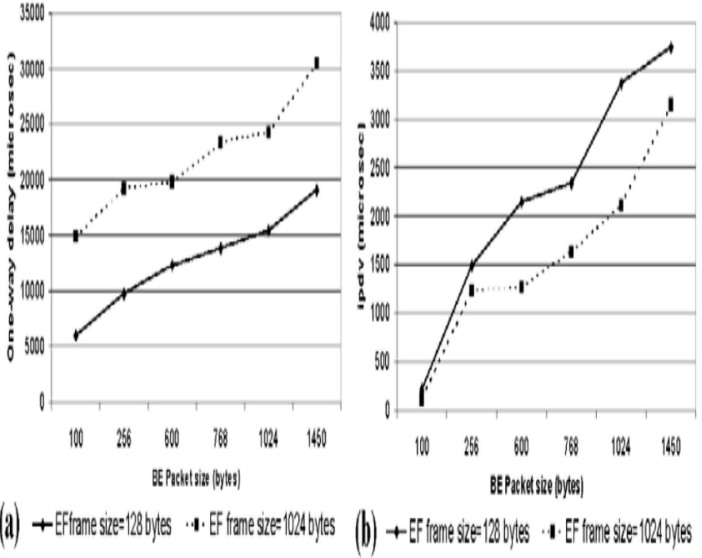

Fig. 6. Impact of the BE packets size on the OWD of EF traffic with the $P Q$ scheduler : experiment [3].
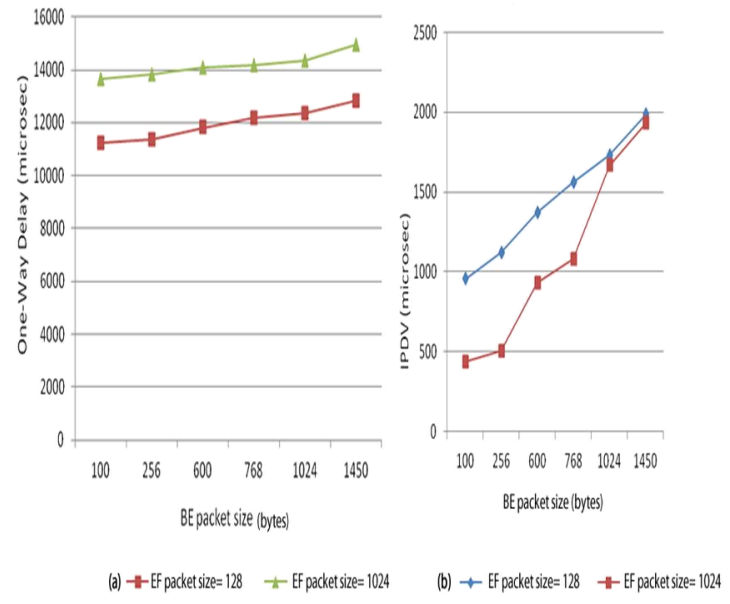

Fig. 7. Impact of the BE packets size on the OWD of $E F$ traffic with the $P Q$ scheduler : simulation

The results show that the OWD and IPDV of EF traffic is proportional to the size of the BE packets and the average OWD increases with the size of the BE packets in the simulation but this growth was low compared to the experiment.

In the PriorityQueueing an EF packet is inserted into the transmission queue as soon as possible. If the interface begins transmitting a packet just before the arrival of the EF packet, it will take L / $\mathrm{Sr}$ before the emission of the priority packet. There is a direct relationship between the delay suffered by the priority flow and the packets size, whether they are priority packets or packets belonging to other queues. 


\section{Scenario $n^{\circ}$ 3: a complete service model}

In this last scenario we have implemented a topology consisting of 3 transmitters of which two WiMAX cell sites and a LAN as described in the figure below:

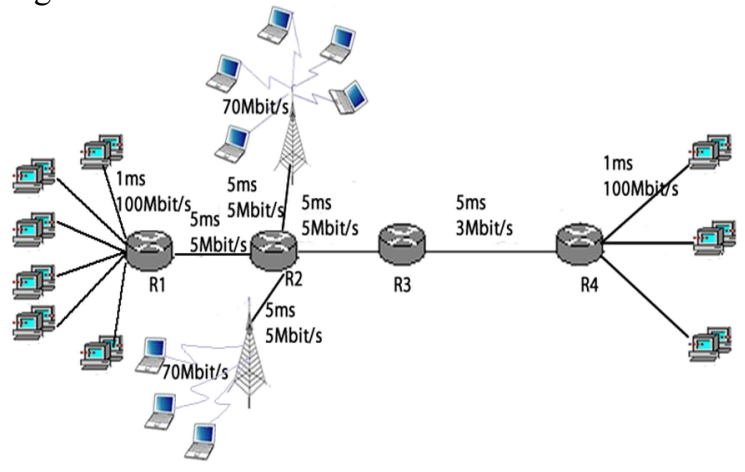

Fig. 8. Architecture scenario $n^{\circ} 3$

On both WiMAX cells we have generated two CRB applications supported by UGS type service flow, 24 Telnet applications and 24 FTP applications respectively mapped in rtPS and nrtPS traffic. Wired stations generate best effort traffic. To analyse the aggregation effects, the measurements have been carried at the router R2. On the scheduling policy at a first level, packages of $\mathrm{AF}$ and $\mathrm{BE}$ classes have been classified into a queue with the WFQ algorithm, the same for those of rtPS, nrtPS and BE services of WiMAX. At the second level we have applied a PQ policy between the queue coming from the WFQ scheduler and the EF class queue in DiffServ or UGS of WiMAX. The configuration of the interface $\mathrm{R} 2$ to $\mathrm{R} 3$ is described in Table III.

Table III: TCS for Interface R1 to R2

\begin{tabular}{|c|c|c|c|c|c|}
\hline $\begin{array}{c}\mathrm{PH} \\
\text { B }\end{array}$ & $\begin{array}{l}\text { Traff } \\
\text { ic }\end{array}$ & $\begin{array}{c}\text { DSC } \\
\mathbf{P}\end{array}$ & $\begin{array}{c}\text { Drop } \\
\text { preceden } \\
\text { ce } \\
\text { level } \\
\end{array}$ & Meter & Dropper \\
\hline EF & CBR & 46 & & $\begin{array}{l}\text { Token } \\
\text { bucket } \\
\text { CIR 500 } \\
\text { kbps } \\
\text { CBS } 100 \\
\text { kbps }\end{array}$ & $\begin{array}{l}\text { Delete } \\
\text { non- } \\
\text { complian } \\
\text { t packets }\end{array}$ \\
\hline $\begin{array}{c}\text { AF1 } \\
1 \\
\end{array}$ & $\begin{array}{l}\begin{array}{l}\text { Telne } \\
\mathrm{t}\end{array} \\
\end{array}$ & 10 & 0 & & \multirow{3}{*}{ WRED } \\
\hline $\begin{array}{c}\text { AF1 } \\
2\end{array}$ & FTP & 12 & 1 & \multirow{2}{*}{$\begin{array}{c}\text { TSW2C } \\
\text { M }\end{array}$} & \\
\hline $\begin{array}{c}\text { AF1 } \\
3\end{array}$ & & 14 & 2 & & \\
\hline BE & & 0 & & $\begin{array}{l}\text { Token } \\
\text { bucket } \\
\text { CIR } 700 \\
\text { kbps } \\
\text { CBS } 100 \\
\text { kbps } \\
\end{array}$ & $\begin{array}{l}\text { Eliminati } \\
\text { on on } \\
\text { threshold }\end{array}$ \\
\hline
\end{tabular}

The queue EF is a simple FIFO, the packets compliant to the profile are marked with the DSCP 46 code and the non-compliant packets with the Code 51 and are automatically dropped. La BE queue is managed by a RED mechanism (RandomEarlyDetection) that helps to prevent congestions before they occur, by detecting in advance the filling of the buffers without waiting for the saturation. Packets are rejected in advance causing the source to reduce its speed. A filling threshold, lower than the saturation rejection threshold, serves as a trigger to the mechanism. The $\mathrm{AF}$ queue may use an extension of the previous known WRED mechanism (WeightedRED). In case of congestion the AF13 packets have the highest rejection probability.

The measurements show that the two EF sources packets, once in a knot, suffer no loss and pass into an almost empty queue ( 0 or a packet), the jitter introduced by PQ is less than that caused by other disciplines, but because of the aggregation, the loss rate of $\mathrm{EF}$ flow can reach $15 \%$ for an aggregate composed of 20 individual sources. Considering that the arrival of a burst of EF packets in a router can only grow due to new flow that are generated by each knot, this catastrophic result is rather understandable. The tests have shown that the rate of loss depends on the number of flow into the aggregate and not the EF flow occupancy rate.

For the PHB AF, we have noticed the elimination of $18.85 \%$ of AF13 class packets because of WRED mechanisms to avoid the congestion.

On the best effort class, all the non-compliant packets to the profile have been dropped. For those who are compliant, the $25.74 \%$ of loss are due to the overflow of the queue.

Table IV: Packets Statistics

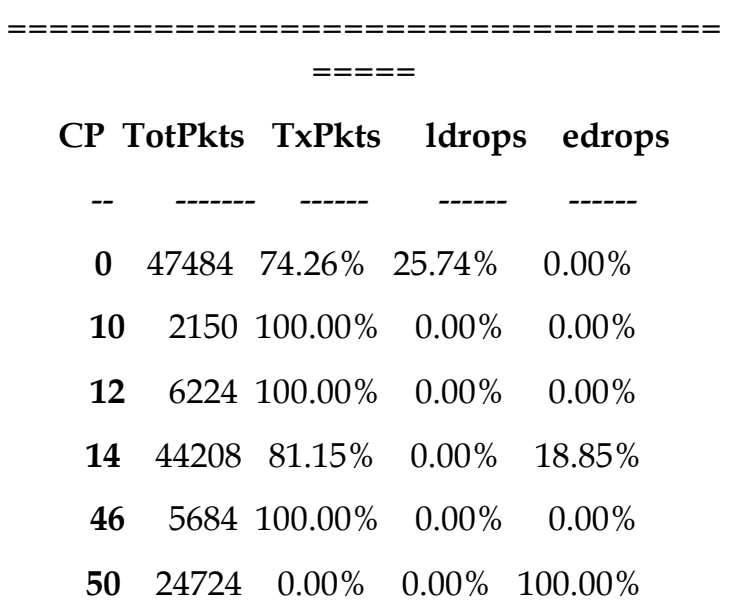

All $\quad 130474 \quad 65.30 \% \quad 9.37 \% \quad 25.34 \%$ 
The Telnet and FTP data flows based on the TCP transport protocol, presents an elastic character through the TCP congestion control mechanism. The speed for TCP is defined as the amount of bytes received by the receiver (except retransmissions) during the transfer time. In the current case, it corresponds to the useful throughput (goodput). Figures 9 and 10 show the throughput results. It shows that the average goodput fluctuates considerably for the $\mathrm{AF}$ class aggregates component. The explanation comes from TCP that reacts by multiplying factor to the loss and by an additive factor to successful transmissions. Indeed, the loss of a packet for TCP, either in profile or out of profile, is detrimental to the average goodput of the flow. TCP responds to the loss irrespective of the priority associated with the packet.

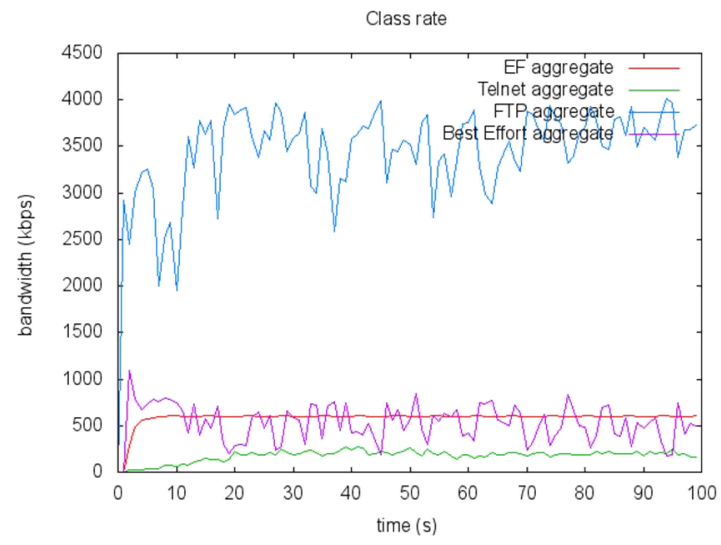

Fig. 9. Goodput by traffic

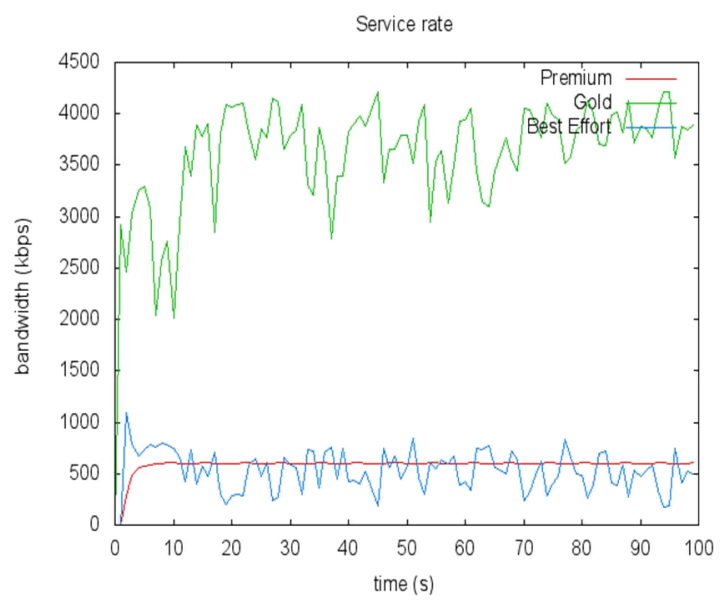

Fig. 10. Goodput by Service Class

\section{CONCLUSION}

In this article we have taken an interest in the size problems of a DiffServ network in WiMAX and in the study of its behaviour.

Concerning the scheduling process, several algorithms can be adopted, but the choice has a great impact on the one-way delay. Our study is mainly focused on WeightedFair Queuing and Priority Queuing. PQ ensures a jitter below WFQ. Under WFQ, a moderate overestimation of service rate optimizes the rate of packets loss, while the impact on the variation of the OWD is less relevant.

Moreover the size of the queue, and the packets size of the UGS traffic are basic parameters that need adjustments so as to preserve the UGS traffic as much as possible from the impact of other aggregates.

The simulation has its limitations; it would be much wiser to deepen studies by experiments.

\section{REFERENCES}

[1] IEEE Std 802.16, "IEEE Standard for Local and metropolitan area networks. Part 16: Air Interface for Fixed Broadband Wireless Access Systems", October 2004.

[2] O. Halidou and R. Mrabet, " End to end IP over IEEE 802.16 QoS framework", International Conference on Sciences of Electronics, Technologies of Information and Telecommunications (SETIT 2012), Sousse,Tunisia, 21/03/2012-24/03/2012, IEEExplore digital library

[3] T.Ferrari (editor), Differentiated Service Experiment: Report; TF-TANT interim report $\begin{array}{llll}\text { Jun } & 99 & - & \text { Sep }\end{array} 99$. http://www.cnaf.infn.it/ ferrari/tfng/ds/delrep1.doc

[4] The Network Simulator NS-2, NIST add-on IEEE 802.16 model (MAC+PHY). September 2006.

[5] RFC2598: An Expedited Forwarding PHB, V. Jacobson and all.

[6] RFC 2679: One-way Delay Metric for IPPM,G.Almes, S.Kalidindi, M.Zekauskas

[7] RFC 3393: IP Packet Delay Variation Metric for IP Performance Metrics (IPPM), C. Demichelis, P. Chimento, November 2002, Proposed Standard. 